This item was submitted to Loughborough's Research Repository by the author.

Items in Figshare are protected by copyright, with all rights reserved, unless otherwise indicated.

\title{
Prediction of air-to-ground communication strength for relay UAV trajectory planner in urban environments
}

PLEASE CITE THE PUBLISHED VERSION

https://doi.org/10.1109/IROS.2017.8206603

PUBLISHER

(C) IEEE

VERSION

AM (Accepted Manuscript)

LICENCE

CC BY-NC-ND 4.0

\section{REPOSITORY RECORD}

Ladosz, Pawel, Hyondong Oh, and Wen-Hua Chen. 2018. "Prediction of Air-to-ground Communication Strength for Relay UAV Trajectory Planner in Urban Environments". figshare.

https://hdl.handle.net/2134/32070. 


\title{
Prediction of Air-to-Ground Communication Strength for Relay UAV Trajectory Planner in Urban Environments
}

\author{
Pawel Ladosz ${ }^{1}$, Hyondong $\mathrm{Oh}^{2}$ and Wen-Hua Chen ${ }^{1}$
}

\begin{abstract}
This paper proposes the use of a learning approach to predict air-to-ground (A2G) communication strength in support of the communication relay mission using UAVs in an urban environment. To plan an efficient relay trajectory, A2G communication link quality needs to be predicted between the UAV and ground nodes. However, due to frequent occlusions by buildings in the urban environment, modelling and predicting communication strength is a difficult task. Thus, a need for learning techniques such as Gaussian Process (GP) arises to learn about inaccuracies in a pre-defined communication model and the effect of line-of-sight obstruction. Two ways of combining GP with a relay trajectory planner are presented: i) scanning the area of interest with the UAV to collect communication strength data first and then using learned data in the trajectory planner and ii) collecting data and running the trajectory planner simultaneously. The performance of both approaches is compared with Monte Carlo simulations. It is shown that the first implementation results in slightly better predictions, however the second one benefits from being able to start the relay mission immediately.
\end{abstract}

\section{INTRODUCTION}

Utilizing wireless communication in an urban environment is challenging due to obstructions and occlusions by buildings, resulting in significant delays and limited range and bandwidth [1]. To facilitate reliable communication between several ground nodes in an urban environment, this work proposes the use of small, low-altitude and low-power (in terms of communication) UAVs as communication relays. UAVs are beneficial for this mission as they can arrive at the mission area rapidly without reliance on roads and existing infrastructure and change their positions with ease in order to adapt to dynamic communication environments.

To plan an efficient UAV relay trajectory, air-to-ground (A2G) communication for arbitrary UAV positions needs to be available. In the past, several approaches were used for communication strength computation and relay trajectory planning. They can be largely divided into the modelbased and measurement-based approaches [2]. In modelbased approaches, communication strength between nodes is calculated by using a pre-defined model with communication parameters which are dependent on the mission environment. Model-based approaches can be further subdivided into

This work was supported by the UK Engineering and Physical Science Research Council (EPSRC) under the Grant EP/J011525/1.

${ }^{1}$ Pawel Ladosz and Wen-Hua Chen are with the Department of Aeronautical and Automotive Engineering, Loughborough University, Loughborough, Leicestershire, LE11 3TU, United Kingdom \{p.ladosz, w. chen\} @lboro.ac.uk

${ }^{2}$ Hyondong Oh is with School of Mechanical and Nuclear Engineering, Ulsan National Institute of Science and Technology (UNIST), Ulsan, Republic of Korea h.oh@unist.ac.kr range-only and channel propagation methods. The rangeonly method was shown in [3], [4] where communication is assumed to be possible only within a certain radius away from the node. The channel propagation method is shown in [5]-[7] where the distance-dependent equation is used to model communication quality. Both range-only and channel propagation approaches suffer from being unadaptable to changes in the communication environment and inaccuracy due to errors in models and unknown noise sources.

To mitigate this issue, measurement-based methods try to use measured communication signal data. They rely on collecting communication data to either create or update the communication strength model in real time. They can be split into gradient following and the learning methods. In the gradient following method such as [8], [9], measurements (e.g. signal-to-noise ratio or received signal strength indication) are used to calculate and follow gradient to better communication strength positions gradually. However, the gradient following method might struggle in urban environments due to numerous and significant signal strength variations caused by buildings and interference. Learning approaches in [10], [11] can mitigate those issues where real communication data is collected and then used to create a correction to a priori communication model. In particular, Gaussian Process (GP) for predicting inaccuracies in a priori A2G communication model was used in [10] for the data ferry mission which was proven to be useful. The UAV collects the data from a fixed ground node and then transmits them to another node outside the normal communication range by physically flying towards it.

Similar to [10], this work uses GP to learn the A2G communication strength for the communication relay mission using UAVs (described in [12]). Unlike ferrying, the relay UAV is to be included in an existing network as a node to provide additional links with the higher bandwidth thus improving communication quality of a team of networked nodes. In this work, a priori communication model consists of a constant path loss and transmission power model, while the GP technique is used to predict the effect of building obstruction and noise sources in the environment on A2G communication strength. Communication strength at arbitrary positions can be predicted by GP and used to plan the efficient relay trajectory for UAVs which maximises communication quality of a team. Main contributions of this paper can be summarised as: i) use of GP to predict a communication map in a complex urban environment and ii) inclusion of GP in the nonlinear model predictive controlbased relay trajectory planning in two different ways. 


\section{PRoblem Definition}

\section{A. Scenario Overview}

A sample scenario considered in this paper is shown in Fig. 1. There are four ground nodes (yellow dots) and one UAV. The trajectory of the UAV is represented with a cyan line. The red lines represent network connections with the minimum communication cost (which will be explained in detail later) and green areas are additional noise patches applied. 30 scenarios were randomly generated with changing the height of buildings, the position of ground nodes and the position and the size of noise patches. The assumption of

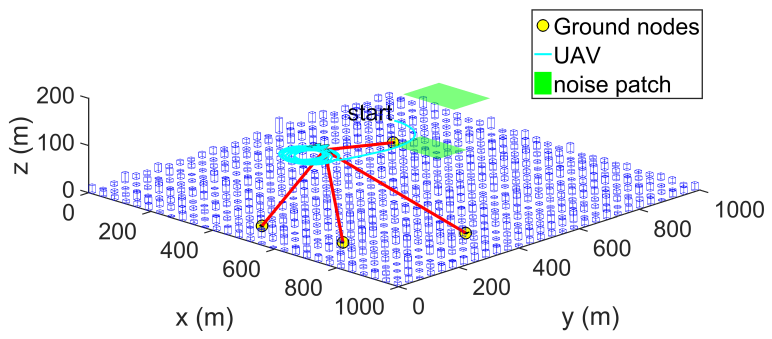

Fig. 1. A sample urban scenario used in this paper.

this work can be listed as follows: 1) Ground node position is known to UAV at all time 2) pathloss and transmitted power components of communication strenght are known 3) Position, sizes and effects of buildings are unknown a priori to UAV 4) Position, sizes and effects of noise sources are unkown a priori to UAV

\section{B. Considered Implementation}

Learning approaches for predicting air-to-ground communication strength are implemented with the relay trajectory planner in two manners: i) scan+NMPC with GP and ii) NMPC with GP. Scan+NMPC with GP starts by performing a scan pattern (shown in Fig. 3) to collect communication strength data for the full environment. This data is used by GP to learn the effect of buildings and other noise sources not considered in a priori communication model. Such a communication map is then used by the relay trajectory planner to guide the UAV on the optimal path. Another implementation, NMPC with GP, takes a slightly different approach. Instead of scanning, the UAV first plans the relay trajectory with the best knowledge it has at that time (i.e. using a priori model initially). Every time it reaches a new position (or periodically), it collects the communication data locally to update its knowledge on the effect of buildings and noise sources using GP. In this way, the UAV saves time for the scan at the expense of the communication prediction quality. Both approaches are described in Fig. 2.

\section{GP-BASED COMMUNiCATion MAP BUILding}

\section{A. Communication Model}

The realistic communication model proposed by [13] is used in this work, which is designed explicitly for aerial platforms. It is based on extensive ray tracing simulations in

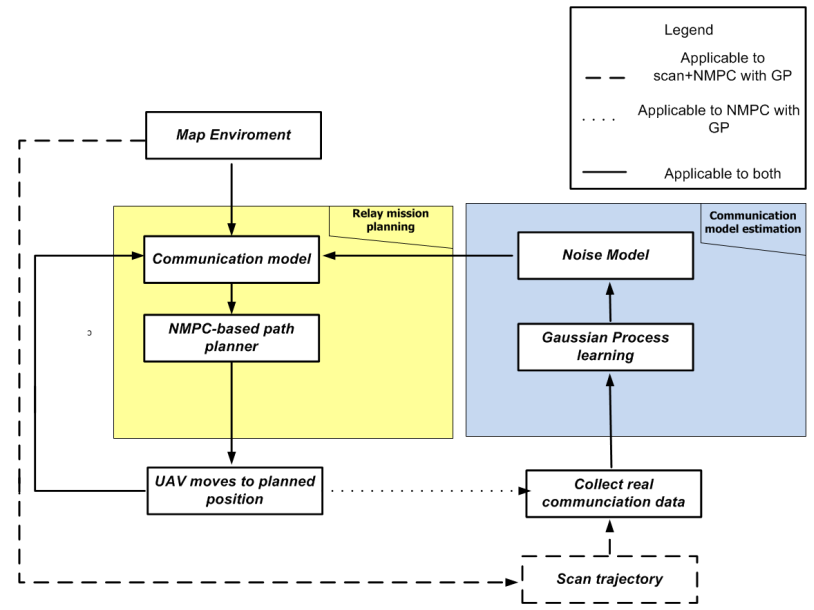

Fig. 2. Overview of the proposed algorithms for the communication relay mission.

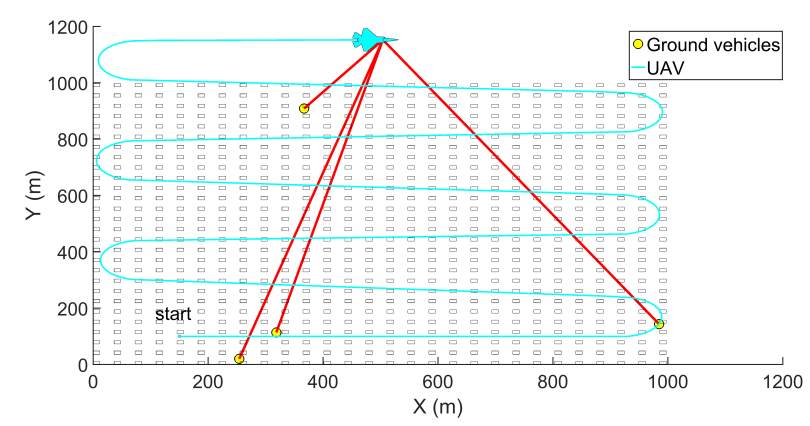

Fig. 3. Pattern for UAV scan on a sample scenario.

four different types of urban environments: Suburban, Urban, Dense Urban and High-rise Urban. The general formulation is defined as [14]:

$$
P_{r, i j}= \begin{cases}P_{t, i}-L_{d B, i j}-\Psi_{L o S}, & \text { if LOS exists } \\ P_{t, i}-L_{d B, i j}-\Psi_{N L o S}, & \text { otherwise }\end{cases}
$$

where $P_{r, i j}$ is a received power strength by node $j$ from node $\mathrm{i}, P_{t, i}$ is transmitted power by node $i, L_{d B, i j}$ is a path loss between nodes $i$ and $j$ and $\Psi_{L o S}$ and $\Psi_{N L O S}$ are shadow fading for when there is line-of-sight (LOS) and NLOS between the UAV and the ground node, respectively. It is worth noting that shadow fading in the model accounts for diffractions and reflections (multipath propagation) only. Path loss can be represented as:

$$
L_{d B, i j}=10 n \log _{10}\left(\frac{4 \pi f_{c} d_{i j}}{c}\right)
$$

where $f_{c}$ is a frequency of transmission, $n$ is a path loss exponent, $d_{i j}$ is distance between ground node $i$ and UAV $j$, and $c$ is a speed of light. Shadow fading is represented with the normal distribution: $\Psi_{L O S} \sim N\left(\mu_{L O S}, \sigma_{L O S}^{2}\right)$ and $\Psi_{N L O S} \sim N\left(\mu_{N L O S}, \sigma_{N L O S}^{2}\right)$ in $\mathrm{dB}$ for LOS and NLOS situation respectively. In [13], it was shown that $\mu_{L O S}$ and $\mu_{N L O S}$ are constant for a given scenario type, whereas $\sigma_{L O S}^{2}$ and $\sigma_{N L O S}^{2}$ can be calculated as:

$$
\sigma_{L O S}^{2}=k_{1} \exp \left(k_{2} \theta_{i j}\right)
$$




$$
\sigma_{N L O S}^{2}=g_{1} \exp \left(g_{2} \theta_{i j}\right)
$$

where $\theta_{i j}$ is elevation angle between ground node $i$ and UAV $j$ and $k_{1}, k_{2}, g_{1}, g_{2}$ are a constant dependent on the environment type. In this work, GP will be used to predict shadow fading component of Eq. (1) as this is mostly dependant on the existence of an obstruction, while path loss and power component will be assumed to be a priori communication model. It is worth noting that other noise sources could also be considered and shadow fading is used as an example of such noises. Additionally, there are extra noise sources added to each scenario to simulate extra unknown interferences. In this work, noises take a very simple form of a rectangular patch. If UAV flies within the patch, the LOS and NLOS (depending on a case) are reduced by a certain amount.

\section{B. Communication Performance Metric}

In this work, global message connectivity (GMC) is used as the communication performance metric to indicate the performance of UAV relay. The GMC is defined as a probability of a message being successfully transmitted to all nodes within the minimum spanning tree (MST) [7]. The MST is defined as a subset of a graph where all nodes are connected to each other but there are no loops, having a minimum (or at least the same as a minimum, as there can be several minimum spanning trees in a single scenario) sum of edge weights [15]. When there are a number of nodes involved in a scenario, the number of possible connections between them in the network could increase significantly. MST solves this problem by considering the most efficient way of connecting all nodes in the network, which facilitate the efficient and reliable sharing of information.

With knowledge of positions of ground node and communication model (consisting of a priori model and GP estimation), the MST can be built. Let the adjacency matrix of the MST be represented as $A^{\prime} \in \mathbb{R}^{(n+m) \times(n+m)}$, where $n$ is the number of UAVs and $m$ is the number of ground nodes, then $A_{i j}^{\prime}=1$ if the link from node $i$ to node $j$ is the part of the MST, and $A_{i j}^{\prime}=0$, otherwise. In the MST, a total communication strength to all nodes (i.e. GMC performance metric $J_{G M C}$ ) can be represented as the sum of all connections within the MST, and the problem is finally formulated to find the position of relay UAVs which maximises $J_{G M C}$ :

$$
\max _{\overline{\mathbf{x}}^{p o s}} J_{G M C}\left(\overline{\mathbf{x}}^{\text {pos }}, \overline{\mathbf{x}}^{g, p o s}\right)=\max _{\overline{\mathbf{x}}^{p o s}} \sum_{i=1}^{n+m} \sum_{j=1}^{n+m} A_{i j}^{\prime} P_{r, i j}
$$

where $\overline{\mathbf{x}}^{\text {pos }} \in \mathbb{R}^{3 \times n}$ and $\overline{\mathbf{x}}^{g, \text { pos }} \in \mathbb{R}^{3 \times m}$ represent the position of UAVs and ground nodes, repectively.

While such a metric has several advantages such as ability to fix many weak connections at once and maximising the number of improved connections in the group, it has some disadvantages. One of key disadvantage of this metric is the fact that it can sometimes ignore very weak connections, to improve a group of already good ones if sum of them would bring bigger benefit than improving weak one. To mitigate that other communication performance metrics could have been used such as worst case connectivity or modified global message connectivity [12]. First one would make UAV improve weakest connection while second one would look to improve a set of connections of strength below pre-specified threshold.

\section{City Model}

To compliment aforementioned communication model same environment model as defined in [13] was used. One of the biggest advantages of this model is that city can be randomly generated from a set of pre-specified parameters. The urban environment model used in that paper was proposed by The International Telecommunication Union (ITU-R) in [16]. The model can be fully characterised by three parameters: $\alpha_{0}, \beta_{0}$ and $\gamma_{0}$ which describe an urban environment in a statistical manner. In particular, $\alpha_{0}$ is a ratio of land cover by buildings to total land area, $\beta_{0}$ is a mean number of building per unit area and $\gamma_{0}$ is a scale factor that describes the buildings heights in Rayleigh probability density function as:

$$
P(h)=\frac{h}{\gamma_{0}^{2}} \exp \left(\frac{-h^{2}}{2 \gamma_{0}^{2}}\right)
$$

where $h$ is building heights in meters. The model considers four environments: (i) Suburban, (ii) Urban, (iii) Dense urban (iv) Highrise Urban. In this paper, suburban environment type is used with $\alpha_{0}=0.1, \beta_{0}=750$ and $\gamma_{0}=8$.

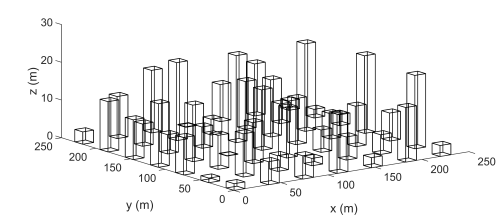

Fig. 4. Sample city generated with parameters: $\alpha_{0}=0.1, \beta_{0}=750$ and $\gamma_{0}=8$.

To fully define the city model not only parameters $\alpha_{0}, \beta_{0}$ and $\gamma_{0}$ needs to be known but also a layout of a city. While cities may follow similar distribution in terms of buildings overall area and sizes, the layout varies significantly from city to city. This paper follows a 'standard city' layout as defined by [13] as shown in Fig. 4.

\section{Gaussian Proccess}

Many previous approaches used a priori model of communication. Such an approach has several disadvantages. The communication environment is likely to be changed by the time the model is used. Additionally, it is possible that additional noise interference is present in the area further reducing the feasibility of pre-computed communication map. In this section, we propose the use of a learning approach to learn positions and effect of unpredicted noise (in this work in the form of shadow fading).

Generally, GP can be formulated as [17]:

$$
f \sim G P\left(\boldsymbol{m}(x), \boldsymbol{k}\left(x^{\prime}, x\right)\right)
$$


where $\boldsymbol{m}(x)$ is a $N \times 1$ vector of mean function, $N$ is a number of collected learning points, and $\boldsymbol{k}\left(x^{\prime}, x\right)$ is a $N \times 1$ vector of covariance function between learning points and the test point. $x$ is a $N \times 4$ training data matrix consisting of the position of UAVs and ground nodes. $x^{\prime}$ is a $N \times 1$ test point vector. Both $\boldsymbol{m}(x)$ and $\boldsymbol{k}\left(x^{\prime}, x\right)$ are characterised by a set of hyperparameters which describe how data is correlated to each other. The number and effect of each hyperparameter are individual to each covariance and mean function type.

The main purpose of training GP is to find a set of hyperparameters such that chosen mean and covariance function fit the learning data best. This is achieved by using log marginal likelihood which can be expressed as:

$$
\begin{aligned}
L & =\log (\mathbf{y} \mid \mathbf{x}, \boldsymbol{\theta})=-\frac{1}{2} \log \left|\boldsymbol{C}_{\boldsymbol{n}}\right| \\
& -\frac{1}{2}(\mathbf{y}-\boldsymbol{m}(x))^{T}\left(\boldsymbol{C}_{\boldsymbol{n}}\right)^{-1}(\mathbf{y}-\boldsymbol{m}(x))-\frac{n}{2} \log (2 \pi)
\end{aligned}
$$

where $\boldsymbol{C}_{\boldsymbol{n}}=\Sigma+\sigma_{n}^{2} I$ in which $\Sigma$ denotes a set of covariance functions of $N \times N$ size with entries $k_{i j}=k\left(x_{i}, x_{j}\right)$ for $i, j=1, \ldots, N$ and $\sigma_{n}^{2}$ is a hyperparmeter responsible for accounting for noisy data, $\mathbf{y}$ is a set of measured communication strength of $N \times 1$ size and $\boldsymbol{\theta}$ is a set of hyperparameters.

Finally, to find hyperparameters using Eq. (8), the conjugate gradients method is used [10]:

$$
\boldsymbol{\theta}^{*}=\underset{\boldsymbol{\theta}}{\arg \max }(L)
$$

Once hyperparameters are computed predicted mean and variance at position $x^{\prime}$ can be found as:

$$
\begin{aligned}
& \mu_{p}\left(x^{\prime}\right)=\boldsymbol{m}\left(x^{\prime}\right)+\boldsymbol{\mu}\left(x^{\prime}\right)+\left(\boldsymbol{C}_{\boldsymbol{n}}{ }^{-1}\left(\boldsymbol{y}-\boldsymbol{m}\left(x^{\prime}\right)\right)\right. \\
& \sigma_{p}\left(x^{\prime}\right)=\boldsymbol{k}\left(x^{\prime}, x^{\prime}\right)-\boldsymbol{k}\left(x, x^{\prime}\right)^{T}\left(\boldsymbol{C}_{\boldsymbol{n}}\right)^{-1} \boldsymbol{k}\left(x, x^{\prime}\right) .
\end{aligned}
$$

It is worth noting that $\sigma_{p}\left(x^{\prime}\right)$ not only represents predicted variance but also uncertainty of prediction.

\section{E. Covariance and Mean Functions}

For GP to work well, best mean and covariance function for a given scenario needs to be chosen. Generally, there are two types of covariance functions: stationary and nonstationary. In this work, a non-stationary covariance function is used as it is applicable to situations when no visible mathematical pattern is present. Due to the random spread of buildings and node positions, this is the case in this work as illustrated in Fig. 5. This figure shows the surface plot of the mean of shadow fading component from Eq. (1) for a sum of four ground nodes and changing positions of the UAV at 150 meters high. In Fig. 5, areas of high mean shadow fading are generally spaced irregularly, thus non-stationary function is more appropriate.

Several non-stationary covariance functions such as neural network, linear, polynomial and squared exponential with spatially varying lengthscale parameters exist. In this work squared exponential with spatially lengthscale parameters covariance function is used as an example of non-stationary

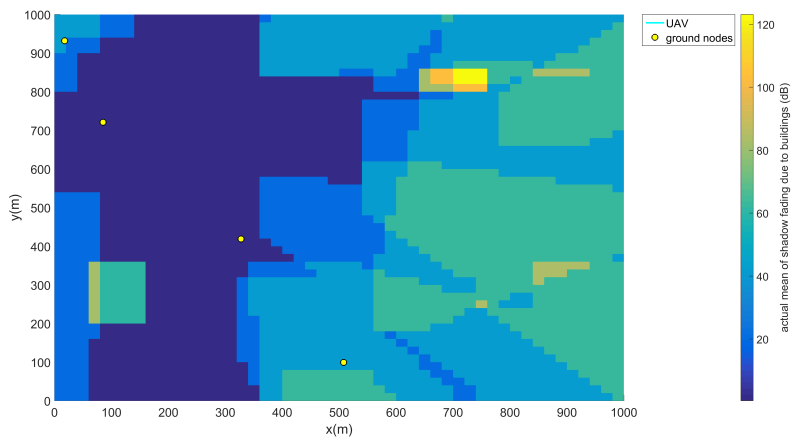

Fig. 5. Shadow fading with $(792,935)$ position of ground node and UAV flying at 150 meters height.

covariance function Squared exponential with spatially varying lengthscale parameters covariance function is formulated as:

$$
k\left(x^{\prime}, x\right)=\sigma_{f}^{2}\left(\frac{a}{b}\right)^{\frac{n}{2}} \exp \left(\frac{\left\|x-x^{\prime}\right\|^{2}}{b}\right)
$$

where $a=2 l(x) l\left(x^{\prime}\right), b=2 l^{2}(x)+l^{2}\left(x^{\prime}\right)$ and $n$ is a number of variables being correlated as defined previously. Finally $l(x)$ and $l\left(x^{\prime}\right)$ are spatially varying lengthscale parameters which are of the same form as a mean function.

The mean function was chosen to best compliment covariance functions. Through experimentation, it was found that constant mean works best with squared exponential with spatially varying lengthscale covariance function in considered scenarios. Such a mean function is denoted as:

$$
m\left(x^{\prime}, x\right)=c
$$

where $c$ is a hyperparameter to optimise.

\section{NMPC-BASED TRAJECTORY PLANNER FOR UAV COMMUNICATION RELAY}

In this section, nonlinear model predictive control (NMPC)-based trajectory planner for UAV communication relay is briefly presented. The NMPC-based trajectory planning approach is chosen for several reasons. First, in the NMPC with GP implementation, the communication model is updated periodically during the mission as new communication measurements arrive. Thus, the NMPC is ideally suited to account for such a change. Second, although stationary nodes are used in this initial proof of concept, an extension with mobile ground nodes is planned in the future. Finally, as the NMPC-based approach only optimises up to a certain length (rather than a full scenario), the problem space is reduced, leading to the less computational time. For more details, readers are referred to [12].

\section{A. UAV Kinematic Model}

This work utilises a simple two-dimensional UAV kinematic model by assuming the UAV speed is constant and the UAV flies at the constant altitude. The UAV model is 
given as:

$$
\left(\begin{array}{c}
\dot{x} \\
\dot{y} \\
\dot{\psi} \\
\dot{\omega}
\end{array}\right)=f\left(\mathbf{x}, u_{\omega}\right)=\left(\begin{array}{c}
v \cos \psi \\
v \sin \psi \\
\omega \\
-\frac{1}{\tau_{\omega}} \omega+\frac{1}{\tau_{\omega}} u_{\omega}
\end{array}\right)
$$

where $\mathbf{x}=\left(\begin{array}{llll}x & y & \psi & \omega\end{array}\right)^{T}$ are the inertial position, heading, speed and yaw rate of the UAV, respectively. $\tau_{\omega}$ is time constant accounting for actuator response delay, which can be determined experimentally for given UAV model. $u_{\omega}$ is a command input in form of turning rate. In this work, the command is constrained by:

$$
\begin{gathered}
\left|u_{\omega}\right| \leq \omega_{\max } \\
\left|u_{\omega, k}-u_{\omega, k-1}\right|=0.1
\end{gathered}
$$

where $k$ is a current time step. The first constraint limits the maximum heading rate of the UAV. The second constraint limits the rate at which heading changes and allows for discretisation of the controller. Both constraints are the result of dynamic limits of a fixed-wing UAV. The UAV model from Eq. (14) is discretised using Euler integration as:

$$
\mathbf{x}_{k+1}=f_{d}\left(\mathbf{x}_{\omega, k}, u_{k}\right)=\mathbf{x}_{k}+T_{s} f\left(\mathbf{x}_{k}, u_{\omega k}\right)
$$

where $\mathbf{x}_{k}=\left(\begin{array}{llll}x_{k} & y_{k} & \psi_{k} & \omega_{k}\end{array}\right)^{T}$ and $T_{s}$ is a sampling time.

\section{B. NMPC-Based Trajectory Planning}

The kinematic model is combined with the NMPC-based trajectory planner to determine the optimal trajectory for UAV communication relay. Such an approach relies on the finite time horizon with the nonlinear model of the UAV. Time horizon defines how far into the future UAV behaviour is predicted. Optimisation is used to determine the best set of control inputs over the horizon length. Only first step is fed into the actual controller and the optimisation procedure repeats for the next time step.

\section{Performance Index}

Dynamic constraints of UAVs are combined with the performance metric defined in Section III-B to create a cost function needed for NMPC trajectory planner formulation as:

$$
J_{d}=\Phi\left(\bar{x}_{N}, \bar{x}_{N}^{g}\right)+\sum_{k=0}^{N-1} L\left(\bar{x}_{k}, \bar{x}_{k}^{g}, u_{\omega, k}\right)
$$

s.t.

$$
\begin{gathered}
\mathbf{x}_{k+1}=f_{d}\left(\mathbf{x}_{k}, u_{\omega, k}\right) \\
\omega_{\min } \leq u_{\omega, k} \leq \omega_{\max } \\
\left|u_{\omega, k}-u_{\omega, k-1}\right|=\Delta u_{\omega}
\end{gathered}
$$

where:

$$
\Phi\left(\overline{\mathbf{x}}_{N}, \overline{\mathbf{x}}_{N}^{g}\right)=p_{c} J_{z}\left(\overline{\mathbf{x}}_{N}^{\text {pos }}, \overline{\mathbf{x}}_{N}^{g, p o s}\right)
$$

$L\left(\overline{\mathbf{x}}_{N}, \overline{\mathbf{x}}_{N}^{g}, u_{\omega, k}\right)=\frac{1}{2}\left[q_{c} J_{G M C}\left(\overline{\mathbf{x}}_{k}^{\text {pos }}, \overline{\mathbf{x}}_{k}^{g, p o s}\right)+r_{v}\left(\frac{u_{\omega, k}}{\omega_{\max }}\right)\right]^{2}$

where $J_{G M C}$ represents the GMC performance index and $\overline{\mathbf{x}}_{k}^{\text {pos }}$ and $\overline{\mathbf{x}}_{k}^{g, p o s}$ are $x$ and $y$ position of UAVs and ground nodes, respectively. $p_{c}, q_{c}$ and $r_{\omega}$ are constant weighting factors. The control sequence $U=\left(u_{\omega, 0}, u_{\omega, 1}, \ldots, u_{\omega, N-1}\right)$ (where $N$ is a horizon step) which optimises the cost function are found by using the custom genetic algorithm (described in more detail in [12]).

Such a formulation allows inclusion of both final position as well as positions at each time step. weighting parameters $p_{c}$ and $q_{c}$ are designed such that contribution of final cost is equal to sum of contributions of costs at each time step along the way. It is worth reiterating that as optimisation is repeated at every sampling time, only first element from the control sequence is used as a control input to the UAV.

\section{Numerical Simulation Results}

To validate the performance of using learning approaches for UAV communication relay, five implementation types are considered as described in Table I. Each implementation had the same constant for all monte carlo simulation starting point (outlined in table III). First two methods were already described in Section II. B: Scan+NMPC with GP and NMPC with GP. Third one assumes full knowledge of the communication map including positions and sizes of buildings and noises and their effect on communication quality with NMPC. Fourth one is loiter without the map information at the starting point where the UAV just turns with a maximum heading rate. Finally, no knowledge of buildings and noise sources is used in the NMPC trajectory planner. In this implementation, the UAV assumes that it flies in an open space with no line-of-sight obstructions. This

\begin{tabular}{|c|c|c|c|c|}
\hline Implementation & \multicolumn{4}{|c|}{ Predicted communication (for MST computation and optimisation) } \\
\hline & \multicolumn{2}{|c|}{ A2G links } & \multicolumn{2}{|c|}{ G2G links } \\
\hline & Path loss & Shadow fading & Path loss & Shadow fading \\
\hline Scan+NMPC with GP & \multirow{5}{*}{ known } & predicted by GP & \multirow{5}{*}{\multicolumn{2}{|c|}{ known }} \\
\hline NMPC with GP & & predicted by GP & & \\
\hline NMPC with map info. & & known & & \\
\hline Loiter without map info. & & $\begin{array}{l}\text { assume all links } \\
\text { are LOS only (for } \\
\text { MST computation) }\end{array}$ & & \\
\hline NMPC without map info. & & $\begin{array}{l}\text { assume all links } \\
\text { are LOS only }\end{array}$ & & \\
\hline
\end{tabular}
is to show the impact of buildings on the communication performance. Note that GP is used only for the prediction of A2G communication links while shadow fading is assumed to be known between ground nodes by assuming that ground nodes stay in given positions for long enough to collect enough data to calculate mean of shadow fading. Numerical

TABLE I

SCENARIOS CONSIDERED IN THIS WORK

simulations are used to compare proposed approaches. Table II shows radio communication parameters [13]. Parameters used for NMPC-based trajectory planner and the urban environment are shown in Table III. 30 Monte Carlo simulations were performed with different building heights, noise source 
TABLE II

RADIO COMMUNICATION PARAMETER

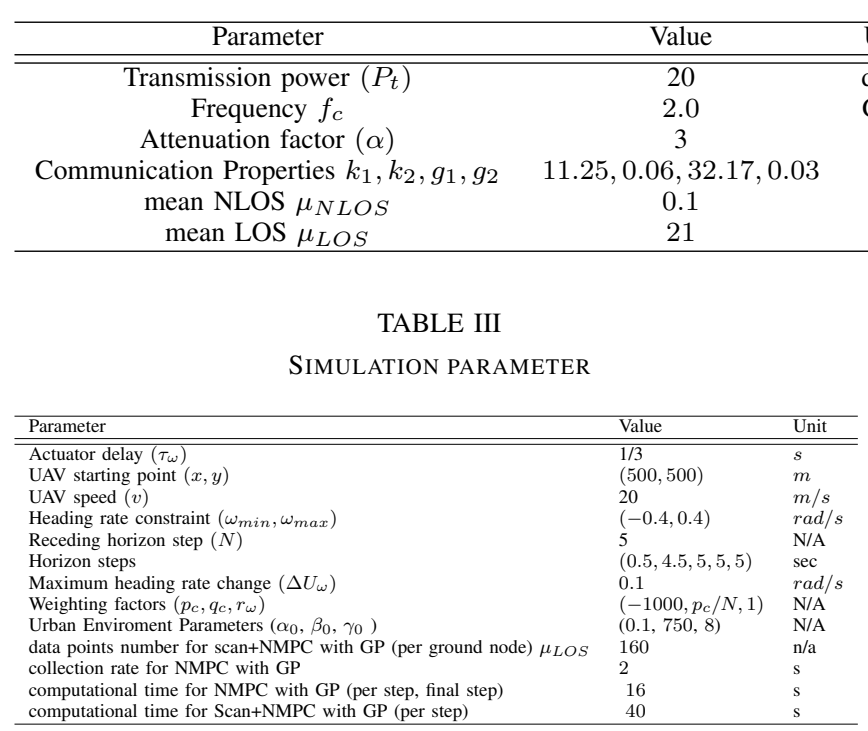

positions and sizes and positions of ground nodes. Note that, for fair comparison, the UAV starting position was the same for all scenarios. This means that, in the case of the scan+NMPC, the UAV returns to the starting position after scan and then performs a relay mission.

From Fig. 6 and Table IV, it is clear that loiter without the map information produces the worst result as the UAV just loiters with around a constant position. NMPC without the map information shows slightly better performance, but still the worst amongst implementations which performs NMPC trajectory planning. Without knowledge of building position and sizes, the UAV does not actively avoid LOS obstruction, thus most of the time all connection within MST are obstructed. NMPC with GP performs worse than scan+NMPC with GP. This implies that depending on user requirements (e.g. whether immediate/timely or better communication is important) different approaches should be chosen. NMPC with the map information performs the best of all implementations, with full knowledge of the communication environment and noise sources. In this ideal situation, the trajectory planner is able to plan the path optimally. A sample trajectory using scan+NMPC with GP is presented in Fig. 7 as an illustrative scenario.

TABLE IV

SUMMARY OF MEAN RESULTS

\begin{tabular}{lc}
\hline Implementation & Communication strength (dB) \\
\hline \hline Scan+NMPC with GP & -943.83 \\
NMPC with GP & -956.14 \\
NMPC with map & -924.48 \\
NMPC without map & -966.89 \\
Loiter without map & -982.27 \\
\hline
\end{tabular}

$\mathrm{p}$

One of the big limitation of of NMPC based control is computational time. As ti can be seen from table [?] real

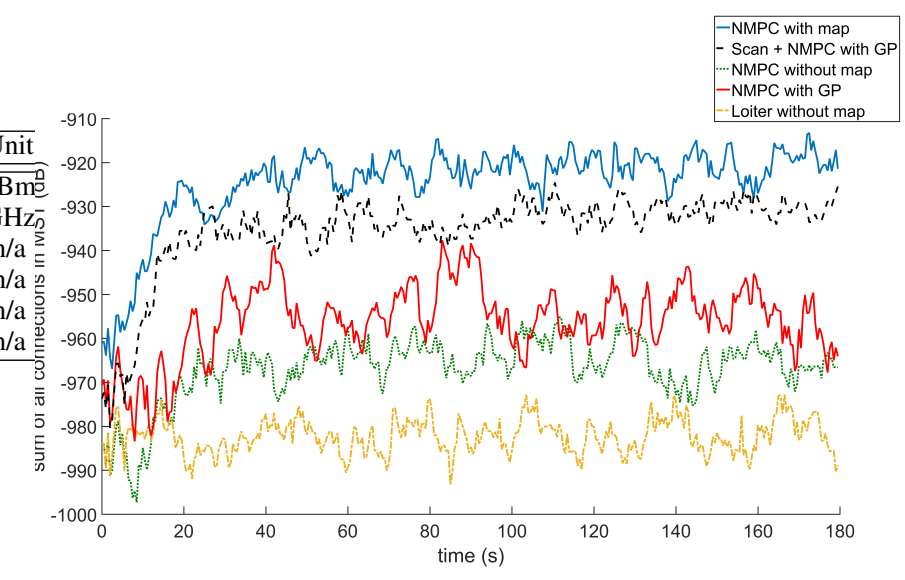

Fig. 6. Comparison between implementations over 30 Monte Carlo simulations.

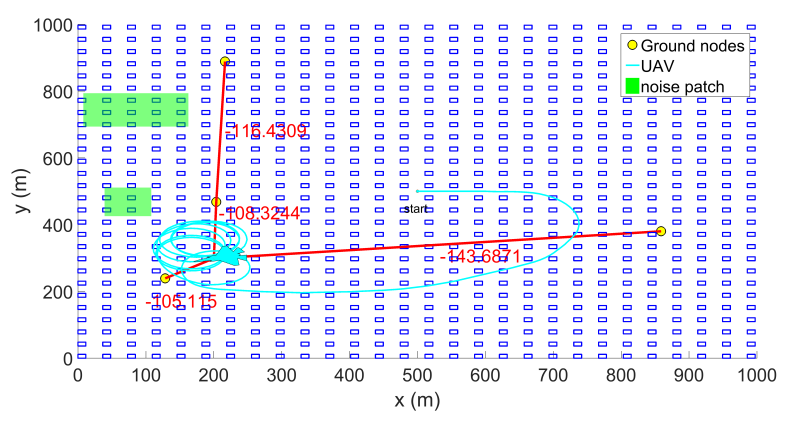

Fig. 7. A sample scenario using scan+NMPC with GP implementation.

time implemantation using matlab is not possible. However there exist several approaches to address this issue such as: i) implement the algorithm in $\mathrm{C} / \mathrm{C}++$. which would reduce computational time signficantly; ii) Increase the size of first step time. This will increase the allowable iteration time but will likely reduce improvment in communciation caused by algorithm; and iii) the number of iterations or population size of the genetic algorithm can be reduced to achieve significant reduction of the computation time. However, this is likely to reduce the quality of solution.

\section{CONClusions AND Future Work}

The use of GP in support of UAV communication relay was proposed. It was shown that creating a communication strength map using GP before the mission shows an advantage over creating communication map while performing the mission. However, collecting data during the mission means that there is a chance that the UAV can consider any instantaneous change in communication strength and adjust its position immediately. Therefore, an appropriate method should be chosen depending on mission environments. Future work includes extending the scenario to include mobile ground nodes and more realistic noise sources and realistic experiment to prove performance of algorithm. Mobile ground node scenario will use exactly same NMPC controller, however 
two changes will have to be made: (i) assumption of lack of complete lack ofknowledge about urban enviroment will be relaxed (ii) assumption about known g2g links will be replaced. More realistic noise sources will be added to better reflect real scenario

This paper adds to existing literature by considering addition of Machine learning to predict communciation performance for UAV relay problem. Additinoally the scenario considered is signficantly more complex than scenarios in the past. This has created additional challenges for machne learning prompting usage of non-stationary covariance function. Finally two different implementation types were considered to show that if possible it is better to scan the area first, however if relay is needed immidately it can be used with some improvements over ignoring urban scenario completely.

\section{REFERENCES}

[1] D. G. Press, "Urban warfare: Options, problems and the future," Janaury 1999. [Online]. Available: http://web.mit.edu/ssp/publications/ conf_series/urbanwarfare/urbanwarfare.html

[2] H.-S. Shin and P. Segui-Gasco, "UAV Swarms: Decision-Making Paradigms," Encyclopedia of Aerospace Engineering, pp. 1-13, 2014.

[3] E. P. D. Freitas, T. Heimfarth, I. F. Netto, C. E. Lino, C. E. Pereira, A. M. Ferreira, F. R. Wagner, and T. Larsson, "UAV relay network to support WSN connectivity," 2010 International Congress on Ultra Modern Telecommunications and Control Systems and Workshops, pp. 309-314, 2010.

[4] A. Agogino, C. HolmesParker, and K. Tumer, "Evolving large scale UAV communication system," Proceedings of the fourteenth international conference on Genetic and evolutionary computation conference - GECCO '12, pp. 1023-1300, 2012. [Online]. Available: http://dl.acm.org/citation.cfm?id=2330163.2330306

[5] D.-T. Ho, E. I. Grotli, P. B. Sujit, T. A. Johansen, and J. B. De Sousa, "Performance evaluation of cooperative relay and Particle Swarm Optimization path planning for UAV and wireless sensor network," 2013 IEEE Globecom Workshops, pp. 1403-1408, 2013.

[6] S. Kim, H. Oh, J. Suk, and A. Tsourdos, "Coordinated trajectory planning for efficient communication relay using multiple UAVs," Control Engineering Practice, vol. 29, pp. 42-49, 2014.

[7] Z. Han, a. L. Swindlehurst, and K. J. R. Liu, "Optimization of MANET Connectivity Via Smart Deployment / Movement of Unmanned Air Vehicles," IEEE Transactions on Vehicular Technology, vol. 58, no. 7, pp. 3533-3546, 2009.

[8] C. Dixon and E. W. Frew, "Optimizing cascaded chains of unmanned aircraft acting as communication relays," IEEE Journal on Selected Areas in Communications, vol. 30, no. 5, pp. 883-898, 2012.

[9] D. J. Lee and K. Andersson, "Hybrid control of long-endurance aerial robotic vehicles for wireless sensor networks," International Journal of Advanced Robotic Systems, vol. 8, no. 2, pp. 101-113, 2011.

[10] A. J. Carfang, N. Wagle, and E. W. Frew, "Improving data ferrying by iteratively learning the radio frequency environment," IEEE International Conference on Intelligent Robots and Systems, pp. 1182-1188, 2014.

[11] J. Fink, N. Michael, A. Kushleyev, and V. Kumar, "Experimental characterization of radio signal propagation in indoor environments with application to estimation and control," 2009 IEEE/RSJ International Conference on Intelligent Robots and Systems, IROS 2009, pp. 28342839, 2009.

[12] P. Ladosz, H. Oh, and W.-H. Chen, "Trajectory planning for communication relay unmanned aerial vehicles in urban dynamic environments," Journal of Intelligent \& Robotic Systems, pp. 1-19, 2017. [Online]. Available: http://dx.doi.org/10.1007/ s10846-017-0484-y

[13] A. Al-Hourani, S. Kandeepan, and A. Jamalipour, "Modeling air-toground path loss for low altitude platforms in urban environments," 2014 IEEE Global Communications Conference, pp. 2898-2904, 2014.

[14] M. Mozaffari, W. Saad, M. Bennis, and M. Debbah, "Efficient Deployment of Multiple Unmanned Aerial Vehicles for Optimal Wireless Coverage," Accepted in the IEEE Communications Letters, pp. 1-4, 2016. [Online]. Available: http://arxiv.org/abs/1606.01962
[15] R. Sedgewick and K. Wayne, "Minimum spanning trees," 2015 [Online]. Available: http://algs4.cs.princeton.edu/43mst/

[16] ITU-R, "Rec. p.1410-2 propagation data and prediction methods for the design of terrestrial broadband millimetric aadio access systems," P Series Radiowave propagation, 2003.

[17] C. E. Rasmussen and C. K. I. Williams, Gaussian Processes for Machine Learning. The MIT Press, 2006. 